\title{
Change Blindness When Viewing Web Pages
}

\author{
Daniel Steffner ${ }^{\mathrm{a}}$ and Bo Schenkman ${ }^{\mathrm{b}^{*}}$ \\ ${ }^{a}$ Stockholm University, Stockholm, Sweden \\ ${ }^{\mathrm{b}}$ Blekinge Institute of Technology, Karlskrona, Sweden and Speech, Hearing and Music, Royal Institute of \\ Technology (KTH), Stockholm, Sweden
}

\begin{abstract}
Change blindness on web pages was studied for 20 participants. The purpose was to find how change blindness appears for web pages, and which changes are easier to detect. The task was to detect if a change had occurred and to show this by the means of the cursor. Rensink's flicker paradigm was used, where four categories of changes were presented. It was easier to detect a change not consisting of a person than one with a person. It was easier to detect a change to the left than to the right. The complexity of the web pages did not appear to have an effect, while large changes were easier to detect than small. The results may indicate that focused attention is differently sensitive for different kinds of changes. They also show that change blindness is a general phenomenon that can be applied to the perception of web pages.
\end{abstract}

Keywords: Change blindness, attention, perception, web pages, reaction time

\section{Introduction}

Change blindness is the phenomenon when a person does not detect that something in the visual field has changed. These changes may be large or minor. Most research has to a large extent focused on how this phenomenon appears in natural situations or when a person looks at a visual scene, e.g. a photo. One of the early studies was done by French [1], who used a discrimination paradigm for these effects. Among the various developments when studying change blindness was the introduction of the flicker paradigm by Rensink, O'Regan and Clark [6], where two scenes are presented in succession, with a blank picture, a mask, put between them for a short period, e.g. $80 \mathrm{msec}$. During this short interval large changes may be made in the second picture, without the observer noticing these. An original picture, $\mathrm{P}$, is shown. It is then followed by a mask which is a gray image in order to hide detection of motion. After the short presentation of the mask, the changed picture, $\mathrm{P}^{\prime}$ is shown, see Figure 1. The whole sequence is then repeated till the change is detected. If the mask was not present the change would be easily detected because of the motion. There is a need for attention demanding mechanisms in order to see the change $[5]$.

\footnotetext{
* Corresponding author. E-mail: bo.schenkman@bth.se
}

A common explanation of change blindness is based on how the visual short term memory functions. Visual information is believed to be transmitted through a filter bottleneck demanding attention to a structure for short visual storage with low capacity. In order for the visual information to be processed to acknowledge a change, there is a demand for a constant viewing of what is viewed [3]. Rensink [4, 5] has proposed the coherence theory that is in accordance with the perceptual characteristics of change blindness.

It is important to distinguish between inattentional blindness and change blindness, see Jensen et al [2]. Inattentional blindness is the failure to notice the existence of an unexpected item, while change blindness is the failure to notice an obvious change. They have similar phenomenologies, viz. a person does not see something obvious, but their etiologies and theoretical implications for vision are different. In this paper, the focus is on change blindness.

In recent studies on change detection, one has made use of presentations or displays closer to real world perception [8]. Besides using more or less artificial stimuli in laboratory conditions, one may also study change blindness in situations in real life, in natural images such as photographic pictures or in role games arranged by a test leader. According to Simons and Rensink [10] the counterintuitive find- 
ings of change blindness in naturalistic conditions strongly support the argument that it is not an artifact, but some general failure to retain or compare information from moment to moment.

One new interaction tool is the Internet with its millions of web pages. Web pages change, some slowly, while others change rapidly. It is therefore of practical and ergonomic interest to see how changes on web pages are detected. Secondly, since a web page constitutes a non-natural artifact, it is of interest to understand how a person perceives and detects changes for such objects. A review of how both inattentional blindness and change detection affect human-computer interface design issues may be found in [12]. We therefore find it important to understand change detection in the realm of the Internet and web pages.

This study had as it aims (1) to investigate change blindness in the context of web pages, when an observer uses web pages, and (2) how different kinds of changes affect the detection.

We chose to study four kinds of changes. (1) Size: Is it more difficult to detect big changes than small ones? (2) Complexity: Is the complexity of the content a factor that will affect the detection? (3) Location: Is the location of the change important for detection. Schenkman and Fukuda [11] found e.g. that observers of web pages preferred objects to the left. (4) Person: Are we more attentive to changes of a person than to a non-person. Our hypotheses were the following: (1) Large changes, (2) changes of simple objects, (3) changes to the left and, (4) changes relating to a person, would all be easier to detect than their opposites.

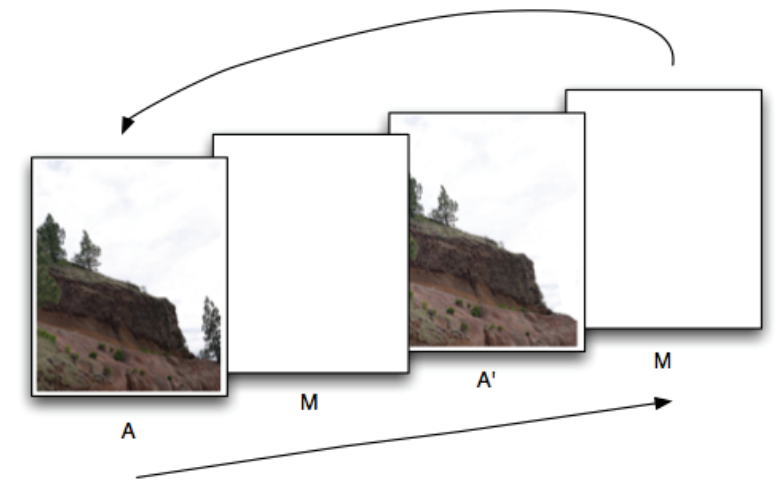

Figure 1. Rensink's flicker paradigm. A mask (M) is shown between the images (A) and (A'). The mask effectively removes the movement normally associated with change. In this case between the image $(\mathrm{A})$ and the altered image $\left(\mathrm{A}^{\prime}\right)$.

\section{Method}

\subsection{Participants}

The flicker method by Rensink et al [5] was used to present different web pages to 20 test persons, all university students, aged 19-42 years, mean $=27.5$ years. All except one person reported that they had good vision, either uncorrected or when corrected with glasses or lenses. The one person who reported that she did not have good vision did, however, used neither glasses nor lenses. One requirement for participation was that they had had been using computers, which all of them had been doing for 4 to 12 years.

The independent variable was the different web pages, where different types of changes, viz. size, complexity, location and person, had been introduced. During up to 60 seconds each pair with blank page, the mask, between them was presented for $240 \mathrm{~ms}+$ $80 \mathrm{~ms}+240 \mathrm{~ms}$. One dependent variable was the detection time for noticing the change. The second dependent variable was the locus of the change that the person had to mark with the cursor.

\subsection{Web pages}

The test program was constituted of 28 computer based presentations or slideshows, where $24 \mathrm{had}$ been designed according to the flicker paradigm by Rensink, O'Regan and Clark [6], as presented in Figure 1. In four of these presentations there was no change in any picture. The 24 sequences were divided into four categories of web pages as to the four hypotheses we wanted to test, thus six sequences in each category. The general layout of the 24 different slideshows is shown in Figure 2. Of these six, three of them were made to belong to one of the tested subcategories, while the other three belonged to the other sub-category. Thus, for the category 'complexity', three of the sequences had changes on simple web pages, while three had changes on complex web pages. The 'simple' web pages had five or fewer web page elements that were distinctly separated with clearly defined borders and margins. The 'complex' web pages had at least nine web page elements that were placed much closer and without distinct separators. For 'location', web page elements with changes were placed either to the left, or right hand side of the web pages. By considering the web pages divided into a grid of three rows and three columns, nine equally sized quadrants of placement were used to 
position the web page elements with changes. The three web pages with changes on the left side each had their web page element with the change in one of the three quadrants in the left column. Analogous principle applied for changes on the right side. For 'person', three had changes on an image of a person, while three classified as 'non-person' consisted of an image of an object or a block of text. For 'size', the changed web page elements categorized as "large" were at least five times the size of those categorized as "small". The presentations were shown to each participant in a random order.

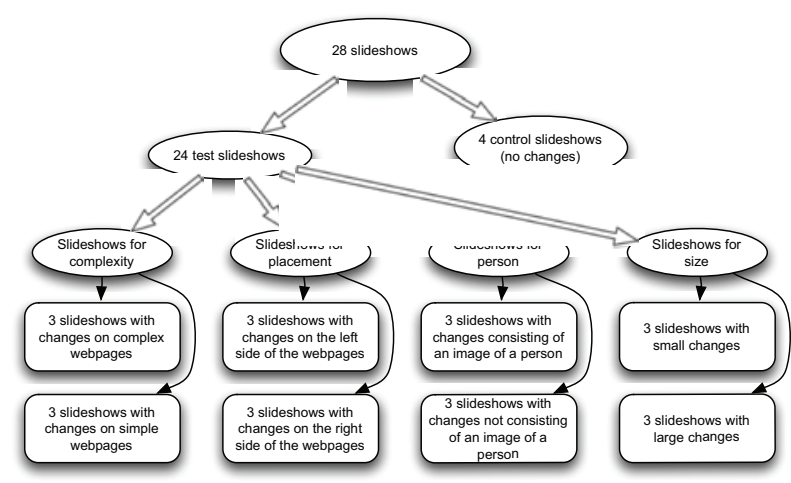

Figure 2. Division of slideshows into categories and sub categories. 28 slideshows were made, 24 for tests and 4 for control. The 24 test slideshows were divided into four categories for the four selected kinds of change. Each of these categories were then divided into two opposing sub categories, each consisting of three different slideshows.

\subsection{Room conditions and equipment}

The image sequences were presented on a 21 inch CRT screen with a resolution of $1024 \times 768$, using a picture frequency of $100 \mathrm{~Hz}$. Luminance at the center of the screen with maximum contrast and lightness was $170 \mathrm{~cd} / \mathrm{m} 2$, when the room illuminance in the horizontal plane was kept at 120 lux, when measured in front of the screen.

\subsection{Procedure}

The participants were informed on paper that they would see a computer presentation with flicker. In some of these there would be a change in the picture. Their task was to detect this eventual change and to mark with the cursor where this change had taken place. They had a time period of $60 \mathrm{~s}$ to detect the change. If they did not succeed within this time duration, the sequence was stopped, and the person was informed if there had been a change that went undetected, or whether there had been no change in that particular sequence. The detection time for the person for each sequence was recorded as was the location where he/she had marked the eventual change.

Before the actual experiment, the participants had a training session of four sequences in order to familiarize them with the equipment, task and procedure. After half of the trials, i.e. after 12 sequences, a short break was had. The total time for completing the experiment varied between the participants, but none lasted longer than 40 minutes. 


\section{Results}

\subsection{Detection times}

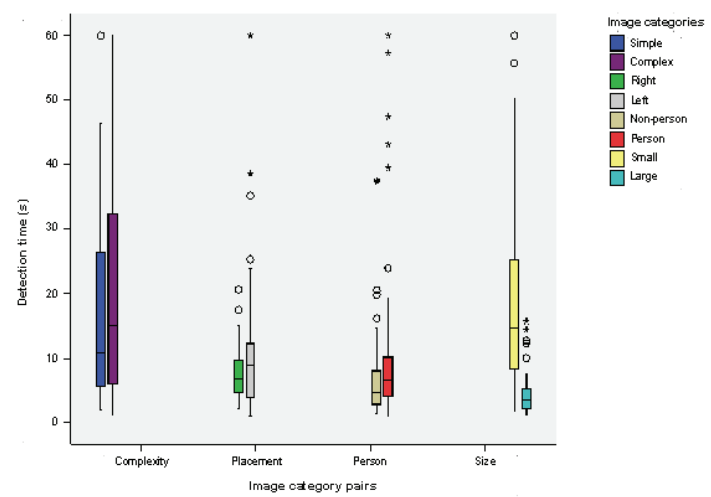

Figure 3. Detection times for the categories and their constituent sub-pairs. Boxes represents standard deviations. o represents outliers, with values within 1.5 and 3 box lengths from the upper quadrille and * represents extreme values with values more than 3 box lengths from the upper quadrille.

The detection times for the different slideshows are shown in Figure 3. The mean detection times varied much between the different pictures. On average over all the participants the detection time was $19.6 \mathrm{~s}, \mathrm{SD}=4.2 \mathrm{~s}$. The fastest individual had a mean detection time of $12.7 \mathrm{~s}$ and the lowest had a mean time of $25.9 \mathrm{~s}$. One-way analysis of variance showed that the changes in the pictures were detected differently fast. Sphericity was not obtained, as shown by Mauchly's test, and the degrees of freedom were therefore corrected. Huyn-Feldt showed significance, $\mathrm{F}(4,462)=15.6, \mathrm{p}<0.001$, as well as the Greenhouse-Geisser test, $F(4,872)=15.6, p<0.001$. Reaction times were not logarithmically transformed, since visual inspection of the data indicated that they appeared to be approximately normally distributed.

For thirteen of the presentations the changes were detected by all participants. In some cases, the observer did not detect the change at all. For five presentations and for another five, one respectively two persons failed to detect the change. For one picture, four people did not detect the change. There were no false positives.

The participants were slowest to detect change in one of the images that had been categorized as simple, constituted of a web page with few objects and with a small change. They were fastest, i.e. having the low- est mean detection time, for a change in a web page having a large change.

Post-hoc analysis of pairwise comparisons with a significance level of $\alpha$ equal to 0.05 showed that changes to the right of the web page, of a non-person object and of big objects were significantly easier to detect than objects on the left, of an image of a person and of small objects, respectively. There was no significant mean difference between web pages that were considered as having simple or complex variations.

\subsection{Search strategies}

The participants had also been asked about their search strategies. The fastest person, 20 years old, had first looked on the whole picture, and if not detecting the change had begun to look systematically from left to right. Ten of the others said that they first had tried to perceive the whole page and then had begun to search in more detail. Eight of the participants did not experience the tests as difficult, which six of them did. Interestingly enough, three participants said the tests were strenuous for the eyes and one of them experienced a slight nausea.

\section{Discussion}

Different kinds of changes on web pages affect how easily they are detected. A little surprising was that changes concerning persons were less easily detected than non-person objects. It is possible that the participants put less weight at images of persons than at other objects. This explanation is supported by findings of $\mathrm{Xu}$ [13], who in studies on face recognition concluded that we are not more predisposed to detect faces than other kinds of objects. However, Ro, Russell and Lavie [7] reached the opposite conclusion. Faces were more easily detected when participants were subjected to the flicker paradigm.

The lack of significance between simple and complex webpages could be caused by an insufficient difference in complexity. It may be that there is a high tolerance to visual clutter before it hinders change detection.

Another unexpected result in the present study is that objects to the left on the screen were more difficult to detect than objects to the right. This is in contrast to what Schenkman and Fukuda [11] found on aesthetic preferences for web pages containing text. This could mean that people do not perceive web 
pages with images in the same way as we perceive web pages with text that we are supposed to read. When changes occur on a web page that has been reloaded by a user, the change may be difficult to detect. Sometimes it might be in the interest of the user that it is emphasized that a change has occurred. In other circumstances, the opposite might apply. People often think that they will notice important events, which reflect a misunderstanding of how attention functions, see Simons and Ambinder [9]. If a person, for example, is to watch a warning display, change blindness may make him/her fail to detect the change. If the change is not within the focus of attention, it may go unnoticed.

The results may also indicate that we do not view web pages in the same manner as we view printed text. The study also gave further evidence that change blindness is a general phenomenon which may occur in many various situations or for various kinds of presentations. However, Simons and Rensink [10] warn for drawing too strong conclusions from studies on change detection to how the visual processes function. For example, a failure to detect a change does not imply the absence of a (mental or visual) representation. Simons and Rensink argue that change blindness studies can only reveal limits of the representation for the conscious perception of dynamic change. We adhere to this warning, but at the same time we believe that the results should be of relevance for web designers and for game applications.

\section{References}

[1] R.S. French, The discrimination of dot patterns as a function of number and average separation of dots. Journal of Experimental Psychology, 26 (1953), 1-19.

[2] M.S. Jensen, R. Yao, R., W.N. Street and D.J. Simons, Change blindness and inattentional blindness, WIREs Cognitive Science, 2 (2011), 529-546.

[3] J.K.. O'Regan, (2007). Change Blindness. http://nivea.psycho.univ-paris5.fr/ECS/ECS-CB.html (201105-23).

[4] R.A. Rensink, Seeing, sensing and scrutinizing, Vision Research, 40 (2000), 1469-1487.

[5] R.A. Rensink, Change detection. Annual Review of Psychology, 53 (2002), 245-277.

[6] R.A. Rensink, J.K. O'Regan, and J.J. Clark, To see or not to see: The need for attention to perceive changes in scenes. Psychological Science, 8 (1997), 368-373

[7] T. Ro, C. Russell, and N. Lavie,. Changing faces: A detection advantage in the flicker paradigm. Psychological Science, 12 (2001), 94-99.

[8] D.J. Simons, Current approaches to change blindness. Visual cognition, 7 (2000), 1-15.

[9] D.J. Simons, and M.S. Ambinder, Change Blindness: Theory and consequences, Current directions in Psychological Research, 14 (2005), 44-48.

[10]D.J. Simons and R.A. Rensink, Change blindness: Past, present and future. Trends in Cognitive Sciences, 9 (2005), 16-20.

[11]B. Schenkman and T. Fukuda, Aesthetic appreciation of webpages as determined by category scales, magnitude estimations and eye movements, in: Proceedings of the International Conference on Affective Human Factors Design, M.G. Helander, M.K. Halimathun, \& T.M. Po, eds., ASEAN Academic Press: London, pp. $309-316$.

[12]D. A. Varakin, D. T. Levin and Roger Fidler, Unseen and Unaware: Implications of Recent Research on Failures of Visual Awareness for Human-Computer Interface Design, Human-Computer Interaction, 19 (2004), 389-342.

[13] Xu, Y, Revisiting the role of the fusiform and occipital face areas in visual expertise, Cerebral Cortex, 15 (2005), 12341242 . 